\title{
Radioterapia paliativa en niños: presentación de un caso clínico y recuerdo de sus indicaciones
}

I. Zapata, R. Magallón, F. Valcárcel, C. de la Fuente, R. Hernanz, A. de la Torre

\section{Resumen}

- Propósito: conocer el papel de la radioterapia como tratamiento paliativo en la patología oncológica pediátrica.

- Caso clínico: se comenta el caso de un niño de 10 años con neuroblastoma estadio IV de inicio que a pesar de sucesivos esquemas de tratamiento por progresión presentó metástasis cerebral única a los tres años del diagnóstico.

- Resultados y conclusiones: revisada la literatura, se puede afirmar que la radioterapia es un tratamiento paliativo eficaz y adecuado cuando nuestro objetivo es controlar síntomas y ofrecer calidad de vida.

Palabras clave:

Paliativo. Metástasis. Radioterapia. Niños.

Oncología, 2004; 27 (2):85-94

Servicio de Oncología Radioterápica.

Hospital Universitario Puerta de Hierro. Madrid 


\section{Zapata y cols.}

\section{Summary}

- Purpose: To find out the role of radiotherapy to palliate metastatic brain tumor symptoms in children.

- Subject and methods: We report a case of neuroblastoma of a 10-year-old child relapsing with symptoms of brain metastasis. The boy received palliative radiotherapy.

- Results and conclusions: Radiation therapy was effective to palliate the brain metastasis symptoms from the treated neuroblastoma. The review of the literature shows that radiotherapy of brain tumor metastases in children is effective to alleviate the symptoms and to afford a better quality of life.

Key words: Palliation. Brain metastasis. Radiotherapy. Children tumors.

\section{Caso clínico}

Niño de 10 años que en diciembre de 2000, tras presentar clínica de dolor en miembro inferior derecho es diagnosticado de neuroblastoma de localización bilateral estadio IV por afectación de médula ósea y captaciones patológicas en gammagrafía ósea (columna dorsal y cadera izquierda). Siguiendo el protocolo SEOP NB-99, inició tratamiento poliquimioterápico sin presentar respuesta tras la primera reevaluación, por lo que se le administró una segunda línea de quimioterapia con persistencia tumoral en suprarrenal y en zona paravertebral derecha. En septiembre de 2001 se realizó cirugía de la masa suprarrenal, confirmándose por anatomía patológica neuroblastoma con cambios postquimioterapia, recibiendo posteriormente tratamiento adyuvante sistémico. En el mes de noviembre se procedió a resecar la masa paravertebral; el informe histológico describe ganglioneuroma. Continuó esquema de quimioterapia con persistencia pero disminución del volumen tumoral, por lo que fue remitido a nuestro servicio para realizar tratamiento locorregional. A principios de 2002 procedimos a la irradiación de un volumen tumoral con márgenes, mediante planificación tridimensional, con fotones de $15 \mathrm{Mr}$ y cuatro campos hasta alcanzar una dosis de $25.38 \mathrm{~Gy}$. En abril de 2002 realizó trasplante autólogo pero a los siete meses se confirma progresión metastásica recibiendo un segundo trasplante en julio de 2003.

En el mes de noviembre ingresa de urgencia por presentar cuadro de cefalea parietotemporal izquierda refractaria a tratamiento médico y acompañada en los últimos días de vómitos en "escopetazo". Se realiza TC craneal (Fig. 1) objetivándose lesión hiperintensa en lóbulo frontal izquierdo de $4 \mathrm{~cm}$ de diámetro con ede-

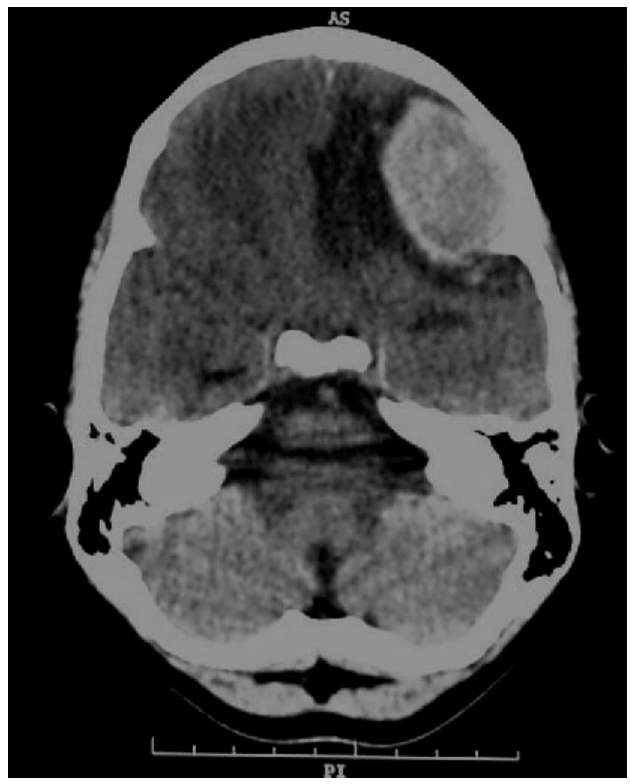

Fig. 1.

ma perilesional importante y desplazamiento de línea media con herniación, compresión del ventrículo lateral izquierdo y sospecha de sangrado acompañante. Nos remiten al paciente tras instaurar tratamiento de soporte y medidas antiedema que no fueron efectivas. De forma urgente inició tratamiento de radioterapia sobre un volumen holocraneal, empleando telecobaltoterapia y dos campos laterales, con un fraccionamiento de $300 \mathrm{cG} /$ día hasta alcanzar una dosis total de $21 \mathrm{~Gy}$. La respuesta al tratamiento fue buena, presentando a los cuatro días de iniciar la irradiación mejoría del nivel de conciencia con desaparición de las náuseas y vómitos y control de la cefalea. 


\section{Introducción}

Los cuidados paliativos en niños, al igual que sucede con los adultos, exigen un manejo multidisciplinar en el que especialistas de distintas áreas deben ofrecer alternativas terapéuticas, no sólo para controlar el dolor sino también otros síntomas asociados a la enfermedad. A diferencia de los adultos, en pediatría es difícil definir un tratamiento como paliativo ya que en muchas situaciones de progresión de enfermedad o de múltiples recidivas se ofrecen varias líneas de actuación, en un intento de rescate aun sabiendo que no se va a controlar ni a modificar su supervivencia. En este contexto de control de síntomas, la radioterapia (RT) puede ser muy eficaz como tratamiento local cuando se pretende tratar la sintomatología producida por metástasis óseas o viscerales, o por efecto directo de la masa tumoral. La radioterapia constituye un tratamiento que a lo largo de la historia se ha intentado excluir en el manejo terapéutico de las enfermedades infantiles por las secuelas que puede producir. En la actualidad, se evita la irradiación en la medida de lo posible y se plantea como alternativa terapéutica de forma restrictiva y con indicaciones muy precisas. Las situaciones paliativas en las que se puede considerar la radioterapia, son las recidivas tumorales locales, las metástasis a distancia y las urgencias oncológicas consideradas como un subgrupo aparte, pero a su vez relacionado con los dos anteriores.

\section{La radioterapia en recidivas locales}

Tumores como el neuroblastoma, rabdomiosarcoma, tumor de Wilms, sarcoma de Ewing, primitivos neuroectodérmicos o del sistema nervioso central pueden presentar recidivas locales que condicionan un efecto masa que produce a su vez dolor, sangrado, obstrucción o síntomas neurológicos. La irradiación ofrece una alternativa cuando se descarta tratamiento quirúrgico por irresecabilidad o elevado riesgo de complicaciones o no se produce respuesta alguna con el tratamiento sistémico. También puede contemplarse dentro de un esquema combinado, asociada a quimioterapia, con el objetivo de disminuir la masa tumoral y tratar así los síntomas producidos. En ocasiones son tumores que ya han sido radiados previamente dentro del esquema terapéutico inicial, con intención radical y curativa' lo que dificulta técnicamente la reirradiación y obliga a asumir una mayor probabilidad de complicaciones y toxicidad a corto, medio y largo plazo. El tratamiento con radioterapia en estos casos deberá considerar volúmenes reducidos, intentando emplear fraccionamientos estándar pero a la vez variables en función de los volúmenes a tratar, del estado general del niño y de la expectativa de vida y también teniendo en cuenta la dosis de tolerancia en órganos críticos, empleando para ello los histogramas dosis-volumen (HDV).

El neuroblastoma es el tumor abdominal más frecuente en niños, que se origina en la médula adrenal $(38 \%)$ o en otras localizaciones paraespinales donde existe tejido nervioso simpático. Las dosis iniciales de RT cuando está indicada, varían según las series pero oscilan entre los 12-36 Gy en función del grupo de riesgo y la edad. La reirradiación en el neuroblastoma se considera de forma individualizada y los efectos secundarios más importantes a tener en cuenta son las deformidades en columna por alteración del crecimiento óseo y de la musculatura paravertebral (que se minimizan incluyendo todo el cuerpo vertebral en el volumen de tratamiento) y la toxicidad renal con alteraciones en el aclaramiento de creatinina.

El tumor de Wilms o nefroblastoma es un tumor maligno embrionario renal que puede asociarse a otras alteraciones. Es un tumor curable con una supervivencia a los 4 años del diagnóstico que supera el $90 \%$. Forma parte también de los tumores considerados como radiosensibles, por lo que los protocolos de actuación contemplan la radioterapia (dosis de 10-20 Gy) en el esquema de tratamiento de enfermedad avanzada o desfavorable por histología ${ }^{2,3}$ y algunos autores hacen referencia a la reirradiación en recidivas ${ }^{4}$ donde la tasa de supervivencia a dos años, tras la recidiva local, no supera el $43 \%$. Se deben vigilar los efectos secundarios en hígado, riñón, gónadas, cuerpos vertebrales y corazón por ser órganos en riesgo en el campo de irradiación y por estar sometidos, a su vez, a la toxicidad de algunas de las drogas empleadas.

Dentro de este grupo de recidivas locales se mencionan las recidivas de tumores cerebrales (meduloblastomas, gliomas, ependimomas...) en las que con el desarrollo de técnicas especiales de planificación "3 $D^{\prime \prime}$ o de radioterapia estereotáxica, tanto fraccionada como de dosis única, se pueden realizar reirradiaciones con una menor morbilidad, no sólo en el contexto de tratamiento paliativo sino también en un intento radical. En esta línea se han publicado varios trabajos que consideran volúmenes reducidos, con limitación de dosis por órganos críticos como son el tronco, médula, vía óptica, etc. no superando los 20-30 Gy y empleando distintos fraccionamientos desde el convencional, hasta el hipo o hiperfraccionamiento ${ }^{5}$. Los objetivos fundamentales, en estos casos, son conseguir estabilizar la enfermedad y paliar síntomas asumiendo un incremento de efectos adversos atribuibles a la reirradiación que puede elevarse hasta un $30 \%$, con un riesgo de 


\section{Zapata y cols.}

radionecrosis del 9-10\%. Los beneficios en supervivencia y paliación han sido modestos, con resultados de supervivencia global de 8.3 meses $^{6}$.

\section{Radioterapia en las metástasis}

Las metástasis sintomáticas constituyen una de las indicaciones menos discutibles y probablemente más eficaces del tratamiento local con radioterapia.

El neuroblastoma presenta una elevada capacidad de diseminación (60-70\%) con afectación de huesos, pulmón, hígado, médula ósea e incluso piel con aparición de metástasis subcutáneas. Existe un subgrupo de enfermedad metastásica, el estadio IV-S (niños menores de un año con tumor primario localizado y diseminación limitada a hígado, piel y/o médula ósea en un porcentaje menor al 10\%) de muy buen pronóstico, con una elevada supervivencia en el que se ha descrito regresión espontánea y que parece tener una explicación relacionada con las variables biológicas y factores genéticos del tumor ${ }^{7}$. La irradiación de metástasis se contempla de forma individualizada y sobre todo en los considerados de alto riesgo, que presentan muy mal pronóstico y en los que las alternativas terapéuticas pasan por distintos ensayos clínicos (empleo de Ac monoclonales, Estroncio-898, 131 I-MIBG ${ }^{9}$ ).

El sarcoma de Ewing es el segundo tumor óseo más frecuente en niños y forma parte del grupo de los tumores considerados radiosensibles. Aproximadamente el $20-30 \%$ presentan metástasis al diagnóstico, siendo las localizaciones más frecuentes el pulmón, hueso y médula ósea y más rara la afectación ganglionar o del sistema nervioso central ${ }^{10}$. Aunque el pronóstico en estos casos es pobre, se ha observado que con terapia intensiva la supervivencia es mayor cuando la diseminación es exclusivamente pulmonar (tasa de curación del $30 \%$ ) que si las metástasis son óseas $(25 \%)$ o se ha producido infiltración medular (20\%). Hay estudios en los que se ha analizado el uso de quimioterapia en altas dosis con o sin irradiación corporal total y con soporte celular, sin obtener mejora en tasas de supervivencia libre ${ }^{11}$. La relativa frecuencia de micrometástasis pulmonares, ha llevado a considerar la utilidad de dosis bajas de radioterapia a nivel pulmonar y se ha llegado a recomendar la irradiación pulmonar completa en todos los pacientes con metástasis pulmonares, incluso si se ha producido respuesta completa a la quimioterapia ${ }^{12}$, aunque esta indicación, en la actualidad, no se aplique.

El $10 \%$ de los tumores de Wilms se presentan en estadio IV por diseminación hematógena, principalmente a pulmón, hígado, hueso y cerebro. La existencia de nódulos pulmonares en la tomografía computarizada
(TC), no es indicación de radioterapia pulmonar total ${ }^{13}$ y sólo se contempla si son visibles en la radiografía de tórax, por lo que el esquema de tratamiento en enfermedad metastásica incluye nefrectomía con linfadenectomía, más/menos radioterapia abdominal con o sin irradiación pulmonar bilateral y quimioterapia posterior.

\section{Metástasis cerebrales}

La diseminación en sistema nervioso central no es muy frecuente en los niños y suele presentarse en estadios muy avanzados. A diferencia de lo que sucede en adultos, la incidencia de diseminación leptomeníngea supera a la de las metástasis cerebrales intraparenquimatosas, probablemente porque los primarios más frecuentes son las neoplasias hematológicas y dentro de ellas las leucemias. La afectación neurológica en el grupo de las leucemias sólo es de un 3\% al diagnóstico, alcanzando cifras del $75 \%$ si no se realiza profilaxis y bajando a un $15 \%$ con la administración del tratamiento profiláctico. La segunda causa la constituyen los tumores cerebrales y entre ellos los tumores primitivos neuroectodérmicos (TPNE) que representan el 33\% y que son seguidos, en menor frecuencia, por melanomas, tumores germinales, sarcomas, linfomas, neuroblastomas ${ }^{14,15}$ y tumor de Wilms.

La sintomatología que se produce con las metástasis cerebrales varía según la localización, pero la más frecuente suele ser cefalea, náuseas, vómitos, mareo, ataxia, crisis convulsivas y si hay una clara focalidad, paresias o alteraciones en la sensibilidad. Si aparecen alteraciones visuales, hipoacusia, parálisis facial o alteraciones en la deglución, se sospechará afectación de pares craneales y cuando la lesión es espinal el niño presentará dolor en columna, irradiado o no, parestesias, disminución de fuerza en extremidades $y / 0$ alteración en el control de esfínteres. El diagnóstico correcto se basará en pruebas de imagen como la TC o resonancia magnética (RM) y en la punción lumbar para el análisis del líquido cefalorraquídeo (LCR) donde se puede conocer la citología y la existencia o no de marcadores tumorales. La RM supera en sensibilidad a la TC al detectar lesiones cerebrales que por escáner no son visibles, aún con el empleo de contraste y es la prueba de elección cuando hay que visualizar médula espinal y canal raquídeo. Dentro de las posibilidades terapéuticas se encuentra el tratamiento médico sintomático, con medidas antiedema (corticoides, manitol), la cirugía (colocación de válvula de drenaje, resección de la metástasis total o parcialmente para descomprimir órganos vitales), la administración de quimioterapia intratecal y el tratamiento con radiotera- 


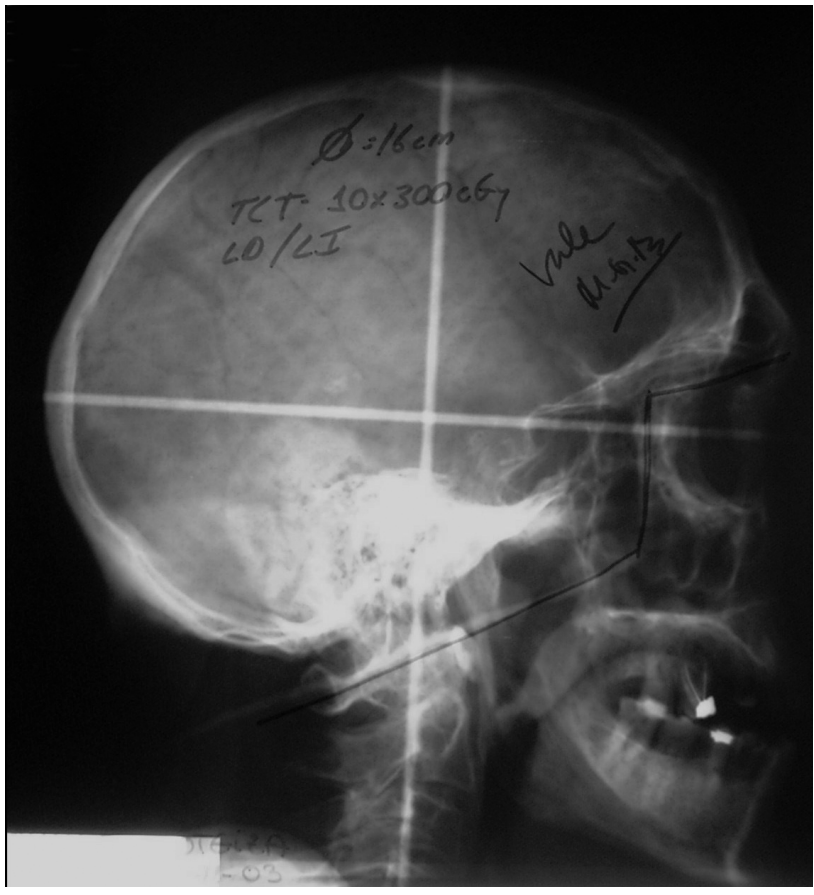

Fig. 2.

pia. La irradiación paliativa no sólo ofrece un control sobre la o las metástasis sintomáticas, sino que también es capaz de evitar la aparición de nuevas lesiones. Lo habitual es incluir todo el volumen cerebral empleando un campo holocraneal (Fig. 2), con un margen adecuado en la base del cráneo para cubrir los lóbulos temporales en su totalidad y con protección de la órbita para excluir los globos oculares. En cuanto a la dosis, pueden emplearse distintos fraccionamientos equivalentes: 5 sesiones de 400 cGy, 10x300 cGy..., administrándose dosis totales de 20-30 Gy que se administrarán en función del tumor, estado basal del niño y del pronóstico de la enfermedad. En adultos existen múltiples series y varios randomizados, comparando distintos esquemas de fraccionamientos y dosis totales de irradiación para el tratamiento paliativo de metástasis, no encontrando diferencias significativas ni en resultados ni en morbilidad ${ }^{16}$. Los resultados confirman un alto porcentaje de control de síntomas y un incremento en la mediana de supervivencia que puede llegar a duplicarse en relación al tratamiento médico exclusivo. Los efectos adversos agudos que pueden aparecer y que se controlan con tratamiento médico sintomático son cefalea, náuseas y vómitos, malestar general y el "síndrome de somnolencia", con un intervalo de aparición que oscila entre 4 y 8 semanas tras finalizar el tratamiento y que cursa con sueño, náuseas, vértigo, irritabilidad y anorexia; este síndrome de- saparece de forma espontánea pero los síntomas mejoran administrando corticoesteroides. Los efectos tardíos de una irradiación cerebral completa y que aparecen a largo plazo son alteraciones neurocognitivas, endocrinas, neuropatías, radionecrosis, segundos tumores... muchas de ellas ya existentes antes de la irradiación, por el propio tumor o la quimioterapia administrada o potenciadas por la asociación de tratamientos. En muchas ocasiones, estos efectos tienen un intervalo de aparición de meses, de forma que no serán documentadas por el mal pronóstico que a corto plazo presenta la propia enfermedad.

Las metástasis leptomeníngeas ${ }^{17}$ pueden aparecer en leucemias, sobre todo las linfáticas agudas y en tumores del sistema nervioso central al producirse una siembra tumoral a lo largo del neuroeje. En estos casos se ofrece una irradiación de las localizaciones de enfermedad bulky o que son sintomáticas, que además se asociará a un tratamiento de quimioterapia intratecal. Los objetivos son lograr una estabilización de la enfermedad y evitar la progresión neurológica, asumiendo que la expectativa de vida de estos niños no supera los 6 meses. Además del tratamiento con volúmenes restringidos (enfermedad macroscópica 20-35 Gy), podemos realizar una irradiación cráneoespinal, técnicamente muy compleja, en decúbito prono, que consiste en añadir a la RT holocraneal la radioterapia de todo el canal raquídeo mediante campos posteriores directos; se recomiendan dosis de 20-30 Gy con posterior sobredosificación de localizaciones afectas hasta 30-55 Gy. Esta técnica es de elevada morbilidad, con una toxicidad aguda (náuseas y vómitos; aplasia de médula ósea con afectación de las tres series: trombopenia, neutropenia, anemia; alopecia...) que en ocasiones es severa y obliga a interrrumpir o suspender el tratamiento. Como efectos secundarios, a largo plazo, destacar los referidos anteriormente con la irradiación holocraneal así como las alteraciones en el desarrollo intelectual, deformidades óseas, riesgo de mielopatías y neuropatías.

\section{Metástasis óseas}

Dentro de la enfermedad diseminada o metastásica, probablemente las metástasis óseas y de partes blandas constituyen una de las indicaciones más claras de tratamiento paliativo con radioterapia, con resultados de control del dolor que alcanzan cifras del orden del 80-90\%. El objetivo no sólo es el antiálgico, sino también el de controlar el crecimiento de la masa tumoral, fracturas patológicas, alteraciones neurológicas o el síndrome de compresión medular. Los primarios más frecuentes son los neuroblastomas, sarcomas (osteosar- 


\section{Zapata y cols.}

coma, síndrome de Ewing, etc.) y tumor de Wilms y para facilitar el diagnóstico se realizarán pruebas de imagen como radiografías (Rx), GGO, TC, RM o rastreo con MIBG en el caso del neuroblastoma.

El tratamiento del dolor tiene un manejo médico protocolizado según escalones, pero además se pueden ofrecer otras alternativas terapéuticas entre las que se encuentra la irradiación local o el empleo de radiofármacos como el Estroncio $90^{8}$ o el 131IMIBG9, en casos de diseminación generalizada y dentro de ensayos clínicos.

Existen pocas publicaciones que traten este tema, pero dentro de ellas las referidas a las metástasis óseas en neuroblastomas son las más numerosas ${ }^{18-20}$. Todas coinciden en la eficacia de realizar un tratamiento local con radioterapia, a pesar de la corta supervivencia que presentan estos niños (supervivencia mediana de 2-2,5 meses tras el tratamiento) presentando un buen control del dolor y/o masa tumoral. Las dosis y fraccionamientos empleados son también variables y en la mayoría de las ocasiones se modifican en función del estado general del niño y del mal pronóstico que presenta la enfermedad en el momento de realizar el tratamiento: 15x400 cGy, 10x200 cGy, 10x250 cGy, 10x300 cGy

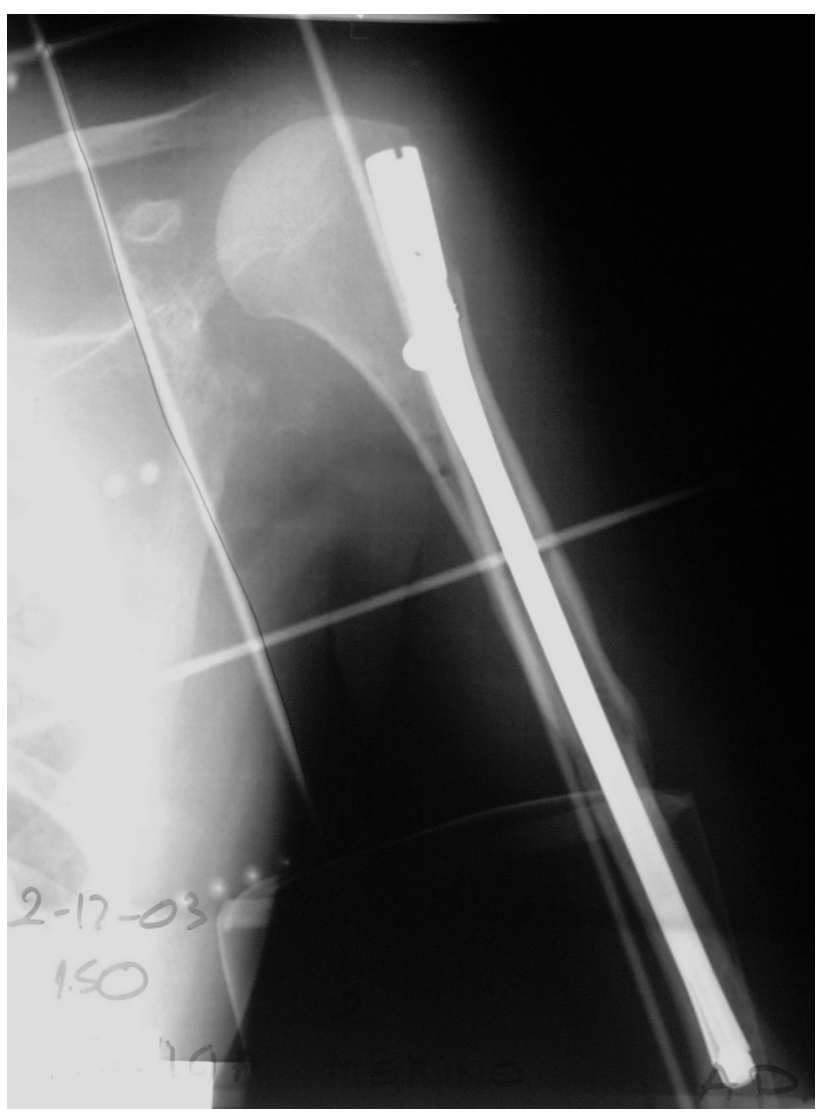

Fig. 3. alcanzando dosis totales que oscilan en un intervalo de 4-30 Gy.

Los efectos adversos de irradiar hueso y partes blandas son principalmente las deformidades, el edema, la rigidez del miembro y/o articulación si está incluida en el volumen de tratamiento, la aplasia de médula ósea si se incluyen huesos planos y en una extensión importante, mielopatías si la afectación es vertebral.... por lo que si es posible se deben proteger articulaciones y órganos críticos y dejar pasillo para favorecer el drenaje de la extremidad afecta.

Una consideración a tener en cuenta y de gran importancia, es que si se ha producido una fractura patológica en un hueso largo o existe un riesgo elevado de producirse, hay que valorar, antes de irradiar, la colocación de una fijación para favorecer la consolidación ósea (Fig. 3).

A diferencia de los adultos, la afectación vertebral no es de las más frecuentes en los niños y es rara, pudiendo eso sí, debutar o progresar como un síndrome de compresión medular.

\section{Metástasis pulmonares}

Aunque la irradiación pulmonar bilateral se contempla dentro del esquema terapéutico de enfermedad diseminada con afectación pulmonar, en algunas patologías como el síndrome de Ewing o tumor de Wilms, a efectos prácticos, la mayoría de los grupos de trabajo intenta evitarla por la elevada toxicidad que puede aparecer: riesgo de neumonitis y neumonía por neumocistis, en muchos casos favorecida también por la asociación de tratamientos (quimioterapia, radioterapia, cirugía).

En el tumor de Wilms ${ }^{21}$ se habla de administrar dosis de 12-15 Gy, valorando incluso un boost de $10 \mathrm{~Gy}$ sobre enfermedad macroscópica y en los sarcomas se recomiendan dosis algo mayores (síndrome de Ewing: 12-21 Gy ${ }^{12)}$. El fraccionamiento empleado es de 150 cGy/día, mediante dos campos anterior y posterior y sobre un volumen pulmonar que incluye desde ápex (por encima de clavículas) hasta las bases pulmonares en su porción más posterior (por debajo de L1), protegiendo hombros y en la medida de lo posible, hígado y riñones.

No hay que olvidar que el manejo terapéutico de las metástasis pulmonares incluye también la administración de quimioterapia y la posibilidad de cirugía ${ }^{22}$.

\section{Metástasis hepáticas}

La irradiación hepática, al igual que sucede con la pulmonar, casi se ha abandonado en las últimas déca- 
das, aunque la literatura y la bibliografía sigue haciendo referencia a esta técnica $7,20,21,23,24$, sobre todo en relación con neuroblastomas y tumores de Wilms. En la mayoría de los casos se valora de forma individualizada y queda reservada a situaciones de rápido crecimiento que produce un compromiso abdominal (vena cava y flujo renal) y respiratorio importante como alternativa a la cirugía y en ocasiones, asociada a quimioterapia. En tumores infantiles lo habitual es administrar dosis bajas de 450-600 cGy, con un fraccionamiento de 150 cGy/día, empleando dos campos, anterior y posterior, sobre todo el volumen hepático y asumiendo el riesgo de efectos secundarios agudos $\mathrm{Co}^{-}$ mo toxicidad hepática aguda que precisa monitorización de enzimas y puede producir trombopenia severa y tardíos que lógicamente no llegarán a presentarse, como hipoplasia pélvica y costal, escoliosis, nefritis, fibrosis hepática y segundos tumores (osteocondromas). La regresión de la hepatomegalia suele ser lenta.

\section{Radioterapia y urgencias oncológicas}

En relación con las patologías previas existen varias situaciones clínicas urgentes que exigen un tratamiento inmediato $y$ en las que la RT ofrece un tratamiento paliativo eficaz: síndrome de vena cava, de compresión medular y leucostasis.

\section{Síndrome de compresión medular}

La compresión medular es infrecuente en niños ${ }^{25}$ (4$5 \%)$ y tiene unas consideraciones propias de incidencia, etiología, mecanismos, clínica y respuesta a tratamiento que la diferencia claramente del síndrome en el adulto. Los tumores que más frecuentemente la producen son el neuroblastoma (8-50\%) por afectación directa, el sarcoma de Ewing (15-28 \%), sarcomas osteogénicos (6-9\%), rabdomiosarcomas (15-28\%), leucemias y linfomas (6-7\%), germinales, Wilms, etc... y puede producirse por distintos mecanismos: metástasis ósea o hematógena (por el espacio epidural utilizando el plexo de Batson), contigüidad, colapso vertebral o por mecanismo neural (extradural, la más frecuente en niños a través de los forámenes intravertebrales).

La localización más frecuente es la torácica y la clínica se caracteriza por aparición de dolor en columna que se asocia a alteraciones neurológicas (disminución de fuerza motora, nivel sensitivo, alteraciones en los reflejos con hiperreflexia y pérdida de control de esfínteres) en un $82-100 \%$ de los casos, rasgo que la diferencia de la sintomatología del adulto donde la aparición de síntomas es más progresiva y no se asocian en un porcentaje tan alto al iniciarse el cuadro clínico.

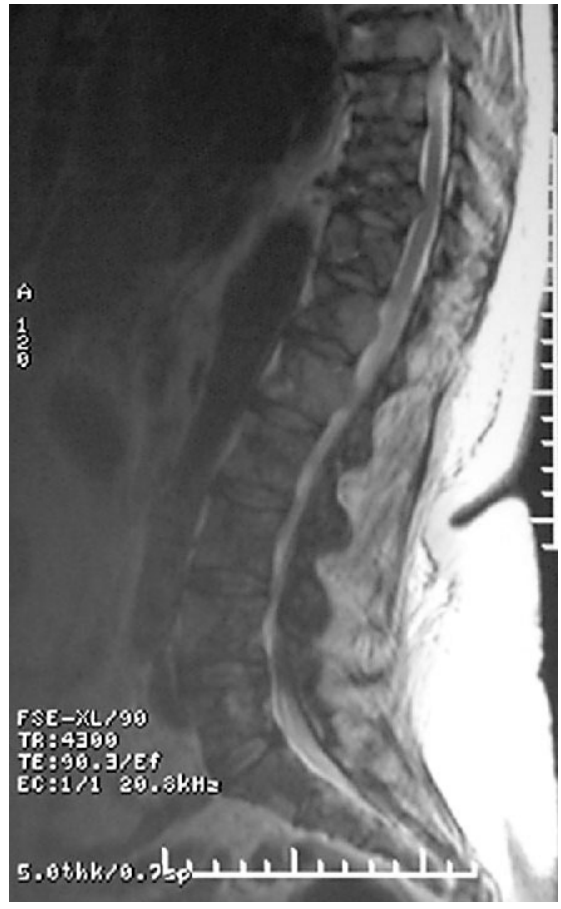

Fig. 4.

Para confirmar el diagnóstico la RM valora el componente de partes blandas y el compromiso del canal medular (Fig. 4). Así como en los adultos se consideran distintas opciones terapéuticas, en los niños la primera opción de tratamiento, aparte del tratamiento médico con esteroides, es la administración de quimioterapia ofreciendo la cirugía (laminectomía) en situaciones de inestabilidad ósea y la radioterapia ${ }^{26,27}$ en aquellos tumores considerados radiosensibles por las posibles secuelas óseas de ambos tratamientos. Los resultados describen un control del dolor en un $70 \%$ (similar en los adultos) con mejoría de la función motora hasta en un $45-60 \%$ (muy superior a la de los adultos donde la incapacidad previa para deambular condiciona los resultados). El volumen de tratamiento suele incluir las vértebras afectas añadiendo 1 ó 2 más por encima y por debajo con una anchura determinada por las apófisis transversas $( \pm 5 \mathrm{~cm})$ y empleando un campo posterior directo con el niño en decúbito prono. Las dosis oscilan entre los 20-30 Gy con fraccionamientos variables: 5x400 cGy, 10x300 cGy...., intentando administrar dosis superiores a los 25 Gy en los linfomas.

\section{Síndrome de vena cava superior}

La afectación de la vena cava ${ }^{28-31}$ por una masa tumoral mediastínica que la infiltra o la comprime, es 


\section{Zapata y cols.}

considerada una urgencia oncológica, que al igual que la compresión medular exige un rápido diagnóstico cuando se sospecha clínicamente: sudoración, plétora y cianosis facial, inyección subconjuntival, edema en "esclavina", incremento de la vascularización sublingual, circulación colateral y posibilidad de aparición de síntomas respiratorios y digestivos con disnea, distress respiratorio y disfagia. Al igual que la compresión medular, es también infrecuente en niños, siendo la primera causa la yatrogénica, relacionada con cirugía cardiovascular o complicaciones de cateterizaciones. Los tumores más frecuentes en niños son las neoplasias hematológicas, principalmente los linfomas no Hodgkin, seguidos de la enfermedad de Hodgkin, las leucemias, neuroblastomas, timomas, teratomas... Mientras que en los adultos puede evolucionar de forma más lenta, en los niños la evolución clínica es rápida, por lo que ante la sospecha se debe realizar una Rx de tórax y un TC torácico que lo confirme para instaurar tratamiento de forma precoz. El tratamiento médico de soporte incluye la administración de corticoides y/o diuréticos, la anticoagulación y el fundamental de elección es la quimioterapia con o sin radioterapia asociada (Fig. 5) que considera dosis bajas (6-7.5 Gy) con un fraccionamiento de 50-200 cGy/sesión. Estas dosis bajas, que no superan los $10 \mathrm{~Gy}$, se consideran suficientes para paliar síntomas. No hay que olvidar la consideración de otros tratamientos locales como la fibrinolisis o la colocación de endoprótesis.

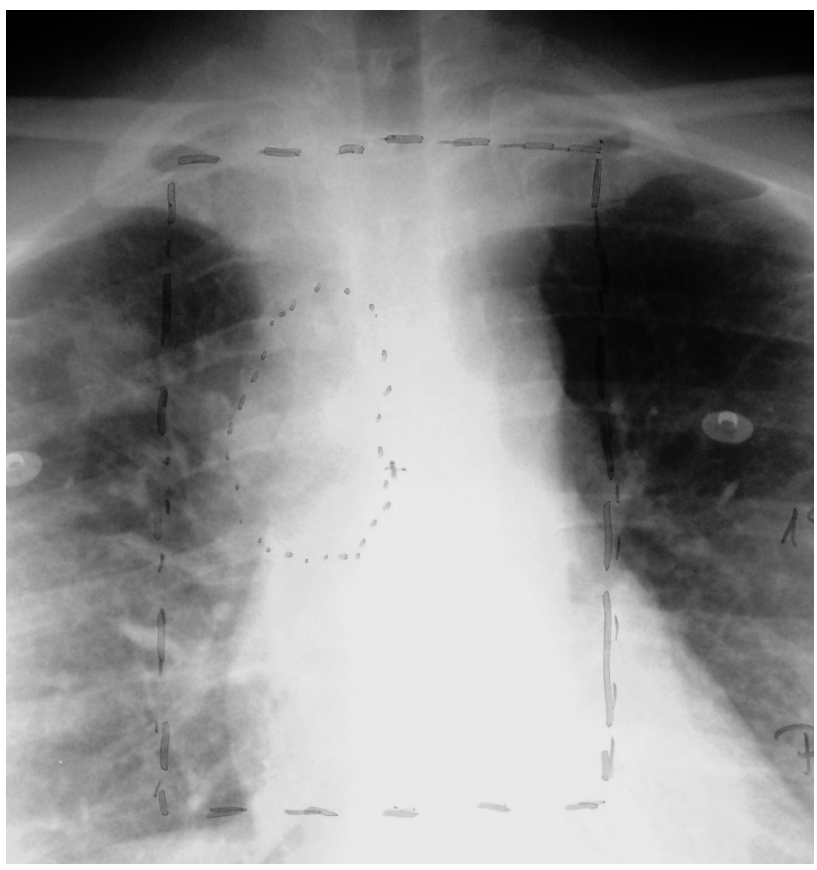

Fig. 5.

\section{Leucostasis}

Son situaciones de hiperleucocitosis ${ }^{32}$ que producen una hiperviscosidad sanguínea por acúmulo de células leucémicas (cifras superiores a 100.000 leucocitos en sangre periférica) que condicionan la aparición de hemorragias, trombosis e infartos. Tiene una incidencia del 9-13\% en niños con leucemia linfática aguda (LLA), y del $5-22 \%$ en leucemias no linfocíticas y mieloides. Los órganos diana que condicionan la aparición de síntomas son el cerebro y el pulmón, cursando con síntomas respiratorios, taquipnea, lisis tumoral, acidosis por hipoxia, etc., que producen en el niño visión borrosa, ataxia, agitación, estupor e incluso coma con una mortalidad elevada. El manejo terapéutico en estos casos tan críticos incluye un tratamiento médico de soporte, fundamental para mantener al niño con vida (hidratación con sueros, alcalinización, alopurinol...) que se acompaña de leucoaféresis (es capaz de corregir viscosidad, alteraciones metabólicas y prevenir el síndrome de lisis tumoral) y de la valoración de un tratamiento con quimioterapia y radioterapia. La radioterapia se considera alternativa cuando las medidas anteriores no han sido eficaces en un intento inmediato de prevenir y evitar la hemorragia realizando una irradiación holocraneal con un fraccionamiento de 150-200 cGy/día hasta alcanzar una dosis de 300-800 cGy (2x150 cGy, 4x200 cGy, incluso 600 cGy en dosis única).

\section{Discusión y conclusiones}

Los tumores pediátricos son, en general, indiferenciados y con un índice de duplicación rápido, lo que hace que respondan bien a los tratamientos sistémicos. Esta característica unida a la tendencia de evitar la radioterapia por la importante morbilidad y efectos secundarios que puede producir sobre los órganos y tejidos que están en pleno crecimiento y desarrollo, ha llevado a no considerarla dentro de las opciones terapéuticas paliativas. La necesidad de incorporar la irradiación en protocolos de tratamiento con intención radical, obteniendo mejoría en control local y supervivencia, debe llevar a reflexionar sobre la utilidad de la radioterapia en enfermedad diseminada cuando la intención ha dejado de ser radical para pasar a ser paliativa, sobre todo en aquellos tumores considerados radiosensibles o con un efecto masa importante. Dentro de los cuidados paliativos de los tumores pediátricos se debe pensar que el tratamiento radioterápico es también adecuado, pero con indicaciones precisas decidiendo en un gran número de casos de manera individualizada. 
Existen pocos estudios y series pediátricas que hagan referencia al tratamiento paliativo, pero hay datos suficientes para afirmar que la radioterapia es eficaz en metástasis sintomáticas ofreciendo resultados en control de síntomas y calidad de vida, por lo que es una alternativa terapéutica que no se debe desestimar.

\author{
Correspondencia: \\ Dra. I. Zapata Paz \\ Servicio de Oncología Radioterápica \\ Hospital Universitario Puerta de Hierro \\ C/ San Martín de Porres, 4 \\ E-28035 Madrid
}

\section{Bibliografía}

1. Matthay KK, Villablanca JG, Seeger RC, et al. Treatment of high-risk neuroblastoma with intensive chemotherapy, radiotherapy, autologous bone marrow transplantation and 13-cis-retinoic acid. Children's Cancer Group. N Eng J Med 1999; 347(16): 1 165-73.

2. Jereb B, Burgers JM, Tournade MF, et al. Radiotherapy in the SIOP nephroblastoma studies: a review. Med Pediatr Oncol 1994; 22 (4):221-7.

3. Green D, Children's Oncology Group: Phase III Multimodality Therapy Based on Histology, Stage, Age, and Tumor Size in Children With Wilms Tumor, Clear Cell Sarcoma of the Kidney, or Rhabdoid Tumors of the Kidney, COG-Q9401, Clinical trial.

4. Miser JS, Tournade MF. The managent of relapsed Wilms tumor. Hematol Oncol Clin North Am 1995; 9: 1287-302.

5. Sheperd SF, Laing RW, Cosgrol VP, et al. Hypofractionated stereotactic radiotherapy in the management of recurrent glioma. Int J Radiat Oncol Biol Phys 1997; 37: 393-8.

6. Bauman GS, Sneed PK, Wara WM, et al. Reirradiation of primary CNS tumors. Int J Radiat Oncol Biol Phys 1996; 36(2):433-41.

7. H. James Nickerson, Katherine K. Matthay, Robert C. Seeger, et al,: Favorable Biology and Outcome of Stage IV-S Neuroblastoma With Supportive Care or Minimal Therapy: A Children's Cancer Group Study. J Clinic Oncol 2000; 18(3):477-86.

8. Charron $M$, Brown $M$, Rowland $P$, et al. Pain palliation with strontium-89 in children with metastatic disease. Med Pediatr Oncol 1996; 26(6):393-6.

9. Westlin JE, Letocha $\mathrm{H}$, JaKobson $A$, et al. Rapid, reproducible pain relief with [131] iodine-meta-iodobenzylguanidine in a boy with diseminated neuroblastoma. Pain 1995; 60(1):111-4.
10. Paulussen M, Ahrens S, Burdach S, et al. Primary metas tatic (stage IV) Ewing tumor: survival analysis of 171 patients from the EICESS studies. European Intergroup Cooperative Ewing Sarcoma Studies. Ann Oncol 1998; 9 (3):275-81.

11. Meyers PA, Krailo MD, Landanyi $M$, et al. High-dose melphalan, etoposide, total-body irradiation and autologous stem-cell reconstitution as consolidation therapy for high-risk Ewing's sarcoma does not improve prognosis. J Clin Oncol 2001; 19(11):2812-20.

12. Spunt SL, McCarville MB, Kun LE, et al. Selective use of whole-lung irradiation for patients with Ewing sarcoma family tumors and pulmonary metastases at the time of diagnosis. J Pediatr Hematol Oncol 2001; 23(2):93-8.

13. Meisel JA, Guthrie KA, Breslow NE, et al. Significance and management of computed tomography detected pulmonary nodules: a report from the National Wilms Tumor Study Group. Int J Radiat Oncol Biol Phys 1999; 44(3):579-85.

14. Kramer K, Kushner B, Heller G, et al. Neuroblastoma metastasic to the central nervous system. The Memorial Sloan-kettering Cancer Center Experience and A Literature Review. Cancer 2001; 91(8):1510-9.

15. Blatt J, Fitz C, Mirro J Jr. Recognition of central nervous system metastases in children with metastasic primary extracranial neuroblastoma. Pediatr Hematol Oncol 1997; 14(3):233-41.

16. Borgelt B, Gelber R, Kramer S, et al. Final results of the first two studies of the Radiation Oncology Therapy Group. Int J Radiat Oncol Biol Phys 1980; 6:1.

17. Chamberlain MC. A review of leptomeningeal metastases in pediatrics. J Child Neurol 1995; 10(3):191-9.

18. Halperin EC, Cox EB. Radiation therapy in the management of neuroblastoma: the Duke University Medical Center experience 1967-1984. Int J Radiat Oncol Biol Phys 1986; 12:1829-37.

19. Deutsch M, Wollman MR. Radiotherapy for metastases to the mandible in children. J Oral Maxillofac Surg 2002; 60(3): 69-71.

20. Paulino AC. Palliative radiotherapy in children with neu roblastoma. Pediatr Hematol Oncol 2003; 20 (2):111-7.

21. Paulino AC. Relapse Wilms tumor: is there a role for radiation therapy? Am J Clin Oncol 2001; 24(4):408-13.

22. Costa Borras E, Ferris i Tortajada J, Jovani Casano C, et al. Surgical treatment of pediatric pulmonary metastases. Cir Pediatr 1998; $11(3): 102-8$.

23. Turek M. Radiation therapy of the liver metastasic disease. Radiol Clin (Basel) 1975; 44(2): 42-5.

24. Blatt J, Dutsch M, Wollman MR. Results of the therapy in stage IV-S neuroblastoma with massive hepatomegaly. Int J Radia Oncol Biol Phys 1987; 13:1467-71.

25. Brian G. Fuller, John D. Heiss, Edward H. Oldfield. Oncologic Emergencies: Spinal Cord Compression. In: Cancer, Principles and Practise Of Oncology, $6^{\text {th }}$ Edition. Vincent T. De Vita, Jr, Samuel Hellman, Steven A. Rosenberg. 2001. 


\section{Zapata y cols.}

26. Katzenstein HM, Kent PM, London WB, et al. Treatment and outcome of 83 children with intraespinal neuroblastoma: the Pediatric Oncology Group experience. J Clin Oncol 2001; 19(4):1047-55.

27. De Bernardi B, Pianca C, Pistamiglio P, et al. Neuroblastoma with symptomatic spinal cord compression at diagnosis: treatment and results with 76 cases. J Clin Oncol 2001; 19(1):183-90.

28. Joachim Yahalom. Oncologic Emergencies: Superior Vena Cava Syndrome. In: Cancer, Principles and Practise Of Oncology, 6th Edition. Vincent T. De Vita,Jr, Samuel Hellman, Steven A. Rosenberg. 2001.
29. Louis S. Constine, Lynda R. Mandell. Lymphomas in Children. In: Principles and Practice of Radiation Oncology, 3th edition. Carlos A. Pérez, Luther W. Brady. 1998.

30. Ingram L, Rivera GK, Shapiro DN. Superior vena cava syndrome associated with childhood malignancy: analysis of 24 cases. Med Pediatr Oncol 1990; 18:476.

31. Yellin A, Mandel M, Rechavi G, et al. Superior Vena Cava Syndrome associated with lymphoma. Am J Dis Child 1992; 146:1060.

32. Leukemia. In: Eduard C. Halperin, Louis S. Constine, Nancy J. Tarbell, Larry E. Kun. Pediatric Radiation Oncology, $3^{\text {th }}$ edition. 1999. 